\title{
Plxnd1 expression in thymocytes regulates their intrathymic migration while that in thymic endothelium impacts medullary topology
}

\author{
Young I. Choi ${ }^{1,2}$, Jonathan S. Duke-Cohan ${ }^{1,2}$, Jing Tan ${ }^{1,3}$, Jingang Gui ${ }^{1,2}$, Manvendra K. Singh $^{4}$, \\ Jonathan A. Epstein ${ }^{4}$ and Ellis L. Reinherz ${ }^{1,2 *}$
}

' Laboratory of Immunobiology, Department of Medical Oncology, Dana-Farber Cancer Institute, Boston, MA, USA

2 Department of Medicine, Harvard Medical School, Boston, MA, USA

${ }^{3}$ Department of Hematology, Affiliated Hospital of North Sichuan Medical College, Sichuan, China

${ }^{4}$ Department of Cell and Developmental Biology, The Cardiovascular Institute, University of Pennsylvania, Philadelphia, PA, USA

\section{Edited by:}

Nick Gascoigne, National University of Singapore, Singapore

Reviewed by:

Kristin Hogquist, University of

Minnesota, USA

Jose Alberola-ila, Oklahoma Medical

Research Foundation, USA

\section{${ }^{*}$ Correspondence:}

Ellis L. Reinherz, Laboratory of Immunobiology, Department of Medical Oncology, Dana-Farber

Cancer Institute, 77 Avenue Louis

Pasteur, HIM 418, Boston, MA 02115,

USA

e-mail: ellis_reinherz@

dfci.harvard.edu
An important role for plexinD1 in thymic development is inferred from studies of germline Plxnd1 knockout (KO) mice where mislocalized CD69+ thymocytes as well as ectopic thymic subcapsular medullary structures were observed. Given embryonic lethality of the Plxnd1 $1^{-/-}$genotype, fetal liver transplantation was employed in these prior analyses. Such embryonic hematopoietic reconstitution may have transferred P/xnd1 KO endothelial and/or epithelial stem cells in addition to Plxnd1 KO lymphoid progenitors, thereby contributing to that phenotype. Here we use Plxnd $7^{\text {flox/flox }}$ mice crossed to $p L c k$-Cre, pKeratin14-Cre, or pTek-Cre transgenic animals to create cell-type specific conditional knockout (CKO) lines involving thymocytes (D1ThyCKO), thymic epithelium (D1EpCKO), and thymic endothelium (D1EnCKO), respectively. These CKOs allowed us to directly assess the role of plexinD1 in each lineage. Loss of plexinD1 expression on double positive (DP) thymocytes leads to their aberrant migration and cortical retention after TCR-mediated positive selection. In contrast, ectopic medulla formation is a consequence of loss of plexinD1 expression on endothelial cells, in turn linked to dysregulation of thymic angiogenesis. D1EpCKO thymi manifest neither abnormality. Collectively, our findings underscore the non-redundant roles for plexinD1 on thymocytes and endothelium, including the dynamic nature of medulla formation resulting from crosstalk between these thymic cellular components.

Keywords: plexind1, thymic development, thymic epithelial cells, angiogenesis, autoimmunity

\section{INTRODUCTION}

The plexins encode large $(\sim 200 \mathrm{kDa})$ transmembrane glycoproteins with domain similarity to the scatter factor receptors encoded by the MET gene family and RON receptor tyrosine kinases (1). There are four plexin families (plexin-A, -B, -C, -D) that function as receptors for multiple subfamilies of semaphorins, sometimes in the context of neuropilin-1, and neuropilin-2, to control cell dissociation or attachment, and repulsion or attraction in a variety of tissue types. Approximately 20 vertebrate semaphorins are known, classified in five distinct subfamilies of secreted and surface-bound proteins (1). In the nervous system, the effects of secreted semaphorin-plexin interaction on axonal pathfinding are generally repulsive (1). The cytoplasmic domain of each plexin contains a GTPase activation domain (GAP) of $\sim 600$ aa encompassing a central Rac- or Rho-like binding motif $(\mathrm{RBD})(2,3)$. These domains have been shown to interact with and regulate the activity of a number of small GTPases including direct binding of Rac1, Rnd1, and RhoD to the RBD and less-well defined sequestration of, or GAP activity against, R-Ras and Rap family members (4-6). The end result is often an effect upon cell motility including an extensive crosstalk with integrin-mediated adhesion (7-9).
In addition to neural development (10), plexinD1 was previously reported to be important in angiogenesis and cardiovascular development $(11,12)$ with consequent effects upon bone vascularization and skeletogenesis $(13,14)$. In addition, lymphoid trafficking including migration of thymocytes (15) and movement of $\mathrm{B}$ cells into germinal centers (16) is regulated by plexinD1. Specifically, with respect to thymocytes, it is the cortical to medullary trafficking of the positively selected double positive (DP) thymocytes which is disrupted in cells lacking plexinD1 (15). Repopulation of irradiated Ly5 congenic mice with fetal liver-derived hematopoietic stem cells from germline knockout (KO) plexinD1 (Plxnd1 $1^{-1-}$ ) mice resulted in developing $\mathrm{CD}^{+} 9^{+} \mathrm{DP}$ thymocytes that exhibited aberrant trafficking following TCR activation (15). In contrast to DP thymocytes arising from wild-type (WT) transplanted fetal liver progenitors, a large fraction of Plxnd1 $1^{-/}$ post-selection $\mathrm{CD} 69^{+} \mathrm{DP}$ thymocytes did not migrate toward the medulla as they further differentiate but rather remained in the cortex. Thymi reconstituted with $P l x n d 1^{-1-}$ hematopoietic progenitors also manifest abnormal ectopic medulla formation, with medullary foci primarily localized to the subcapsular zone.

While recent data showing that the plexinD1 signaling pathway controls both the surface topology of $\beta 1$ integrin nanoclusters 
and individual integrin catch bonds readily account for the thymocyte migratory defect (manuscript submitted), the basis of the ectopic medulla formation is unclear. The role of plexinD1 in determining thymic medullary morphogenesis is complicated by its essential regulation of endothelial cell branching during angiogenesis $(11,12,14)$, a process of critical importance to thymic structure, impacting entry, and egress of lymphoid cells as well as thymic tissue oxygenation (17-19). Sema3E, a soluble secreted molecule whose primary source within the thymus is the medulla, is the physiological ligand of plexinD1 (15). It is through interaction with plexinD1 that sema3E has been demonstrated to regulate vascular endothelial branching in various tissues (20). Developmental analysis of Plxnd1 and Sema3e germline deletion embryos reveals defects in aorta-pulmonary artery large vessel separation (truncus arteriosus) in Plxnd1 $1^{-1-}$ animals but not in the Sema3 $e^{-1-}$ animals, implying a cardiovascular developmental function for plexinD1 distinct from its sema3E ligand (11). Supporting the complexity of plexinD1 function in angiogenesis, VEGF regulates plexinD1 to limit expression to the front of actively sprouting blood vessels where further signaling may or may not occur dependent upon the relative presence of sema3E $(21,22)$.

In this report, we analyze the contributions of plexinD1 in differing cellular thymic compartments that collectively foster thymocyte development. Since germline Plxnd1 deletion is embryonic lethal and thymic reconstitution with stem cells derived from fetal liver may include endothelial progenitors with angiogenic potential as well as other progenitors with epithelial cell potential (23-25), we undertook a systematic analysis of conditional knockout (CKO) mice where cell-specific Plxnd1 deletion allowed development on an otherwise normal B6 background. This strategy permitted us to delineate the functional spheres of operation of plexinD1 within the thymus. Three different $P l x n d 1^{\text {flox/flox }}$ models were developed where transgene expression of Cre recombinase from the pLck promoter (26) in one resulted in deletion of Plxnd1 during thymocyte development, expression of Cre recombinase from the pKeratin14 promoter (27) in a second resulted in deletion of Plxnd1 in thymic epithelial cells (TEC), and expression of Cre recombinase from the pTek promoter (28) in a third resulted in deletion of Plxnd1 in endothelial cells. Using these mouse models, we have determined that loss of plexinD1 expression in thymocytes per se leads to the aberrant migration and cortical retention of $\mathrm{CD} 69^{+} \mathrm{DP}$ cells. On the other hand, ectopic subcapsular medullary formation results from loss of plexinD1 expression on the endothelial cells involved in vascular angiogenesis. Our results provide functional insight into the interplay of angiogenesis, thymocyte maturation, and thymic epithelial cell development in orchestrating $\mathrm{T}$ lineage differentiation.

\section{MATERIALS AND METHODS ANTIBODIES AND REAGENTS}

AnnexinV-FITC, anti-Fc $\gamma$ R (2.4G2), anti-CD69-FITC, antiCD25-PE-Cy7, anti-CD44-APC-Cy7, anti-CD8 $\alpha$-FITC, and antiCD4-APC were obtained from BD-Pharmingen (San Jose, CA, USA). Anti-ESAM-FITC and anti-MHCII (clone M5/114.15.2;
anti-I-A/I-E) was obtained from eBioscience. ER-TR5 was provided by Dr. W. van Ewijk (Leiden University Medical Center, Netherlands), UEA1-FITC was obtained from Sigma-Aldrich. TROMA1 (anti-Keratin8 mAb) clone was obtained from Developmental Studies Hybridoma Bank (Iowa City, IA, USA). Sema3E-Fc was produced as described previously (15); note that the Fc is a mouse $\gamma 2 \mathrm{c}$ isotype. PE-conjugated $\mathrm{F}\left(\mathrm{ab}^{\prime}\right)$ anti-mouse $\gamma 2 \mathrm{c}$ heavy chain and $\mathrm{IgG}_{2 \mathrm{c}}$ control antibody was obtained from Jackson Immunoresearch (West Grove, PA, USA). 145.2C11 mAb was purified directly from hybridoma culture media using Gammabind Plus (GE HealthCare, NJ, USA) and dialyzed against PBS and adjusted to a final concentration of $2 \mathrm{mg} / \mathrm{ml}$.

\section{FLOW CYTOMETRY}

Cell numbers were enumerated using a C-chip hemocytometer (NanoEntek, Korea). In general, single cell suspensions $\left(2 \times 10^{6}\right.$ total) were blocked with $2.4 \mathrm{G} 2 \mathrm{Ab}$ and stained with anti-CD4-APC $\mathrm{mAb}$, anti-CD8 $\alpha$-FITC, anti-CD25-PE-Cy7, anti-CD44-APC-Cy7 $\mathrm{mAb}$, and purified sema3E-Fc $(5 \mu \mathrm{g} / \mathrm{ml})$ for $15 \mathrm{~min}$. After washing with PBS, the cells were stained with anti-mouse $I_{2} G_{2 c}-P E$ to detect bound sema3E-Fc for an additional $15 \mathrm{~min}$. After washing with PBS, the cells were resuspended in PBS and analyzed as described previously (15).

\section{APOPTOSIS ANALYSIS}

One million total thymocytes were stimulated for up to $72 \mathrm{~h}$ on plates pre-coated with anti-CD3 $\varepsilon$ mAb (clone 145.2C11). After incubation, the cells were harvested and stained with anti-CD4APC/anti-CD $8 \alpha-P E / a n t i-T C R \beta$-FITC mAbs and analyzed by flow cytometry. The percentage viable DP thymocytes relative to input at $t=0$ was then determined.

\section{HISTOLOGY, IMMUNOHISTOLOGY, AND CONFOCAL MICROSCOPY}

All the staining procedures were performed as described previously (15). Histological sections were stained using a standard hematoxylin and eosin procedure. In general, adjacent cryosections from thymi of 3- to 4-week-old WT, D1ThyCKO, D1EpCKO, and Sema3e $e^{-l-}$ mice were stained with the anti-CD4-APC/antiCD8 $\alpha$-TRITC mAbs for delineation of cortex from medulla and with anti-CD69-FITC, and MHCII-FITC or UEA1-FITC as required. ER-TR5 (rat IgM) was visualized using anti-rat IgMFITC. Images were obtained using a Zeiss LSM700 confocal laserscanning microscope with $40 \times$ oil lens for objective lens (Carl Zeiss MicroImaging, NY, USA). H\&E staining images were taken using a Nikon Eclipse Ti-S microscope with $4 \times$ dry lens for objective lens (Nikon Instruments, Inc., NY, USA). In every case, images were taken in monochrome to maximize resolution and then digitally recolored prior to merging of images. For identification of Pecam1 on developing endothelial structures, thymi from newborn WT and D1EnCKO mice were collected and fixed in $10 \%$ formalin overnight. Paraffin sections prepared from the fixed thymi were stained with anti-Pecam 1 antibody and, after washing the sections were mounted with DAPI counterstaining the nuclei. In these images, high density nuclear DAPI staining corresponded to cortical regions and lower density staining corresponded with medullary regions. 


\section{MICE AND FETAL THYMI TRANSPLANTATION}

pLck-Cre, pKeratin14-Cre, and pTek-Cre mice were purchased from Jackson Laboratory. The genotyping primers were synthesized by Eurofins. PCR reactions for detection of Cre gene detection were performed as recommended by the Jackson Laboratory. Each Cre strain was backcrossed onto Plxnd1 $1^{\text {flox/flox }}$ background. Since the D1EnCKO mice were neonatal lethal, D1EnCKO or WT fetal thymi were prepared at embryonic day 16.5-17.5 and kept in sterile cold PBS. At this age, WT thymic lobes are fused to form one thymus with two lobes whereas D1EnCKO thymic lobes are not fused. After anesthetizing recipient mice using ketamine and xylazine, fetal thymi were transplanted under the renal capsule through a $0.5 \mathrm{~cm}$ cut in the skin. For WT transplants serving as controls, the complete thymus with two lobes was transplanted whereas for the D1EnCKO transplants, a single lobe was transplanted. After transplantation, the skin portal was closed using sutures. Following surgery, the cages with mice were warmed using hot packs until the mice recovered consciousness. Four weeks later, the thymi developing under the kidney capsule kidney were collected and immersed in OTC for cryosectioning. All the animal experiments were performed under animal protocols approved by Dana-Farber Cancer Institute and Harvard Medical School Animal Care and Use Committee Institutional Review Boards.

\section{WESTERN BLOTTING}

Protein extract from total thymocytes was prepared with IP buffer [1\% NP40, $50 \mathrm{mM}$ Tris- $\mathrm{HCl}, \mathrm{pH} 7.4,150 \mathrm{mM} \mathrm{NaCl}$, plus protease inhibitor cocktail (Roche, Germany)]. Lysate protein concentrations were determined using the BCA Protein Assay Kit (Pierce, IL, USA). Total lysate proteins were separated by SDSPAGE (10\%), transferred to PVDF, blocked overnight using TBS-T containing BSA (1\%), blotted with anti-plexinD1 antisera (SigmaAldrich, MO, USA) followed by anti-rabbit-HRP secondary antibody (Invitrogen, CA, USA), and detection by chemiluminescence (ECL system; Amersham, VA, USA).

\section{STATISTICAL ANALYSIS}

Data are presented as mean values \pm one standard error (SE). P values were calculated using Student's $t$-test.

\section{RESULTS GENERATION OF MICE WITH A THYMOCYTE-SPECIFIC PIxnD1 CKO (D1ThyCKO)}

Given that plexinD1 is operative in multiple developmental processes as described above, the $\mathrm{T}$ lineage autonomous effects of the germline Plxnd1 KO contributing to the previously observed thymic phenotype versus non-T cell lineage cell expression of Plxnd1 remained to be determined (15). Accordingly, a thymocytespecific Plxnd1 CKO mouse strain, termed D1ThyCKO, was created. To this end, $P l x n d 1^{\text {flox/flox }}$ mice generated with $l o x P$ sequences flanking the first exon encoding the transcription initiation site and $5^{\prime}$ sequence encoding the sema domain of the Plxnd1 allele (14) were backcrossed with B6 mice multiple times $(n>10)$ to establish a B6 background. Next, these B6 Plxnd1 flox/flox mice were crossed with $p L c k$-Cre transgenic B6 mice expressing a Cre recombinase under the control of the T cell-specific $p L c k$ promoter. Finally, offspring of these mice were intercrossed to yield the D1ThyCKO animals.

In contrast to germline Plxnd $1^{-1-} \mathrm{KO}$ animals, the D1ThyCKO mice were viable and healthy, despite elimination of plexinD1 expression during $\mathrm{T}$ cell development. Absence of plexinD1 protein was evident both from Western blotting analysis of thymus lysates from these animals (Figure 1A) as well as by FACS analysis of their DP thymocyte surface plexinD1 expression detected by specific binding of sema3E-Fc (Figure 1B). On the other hand, plexinD1 was readily identified in WT samples. Despite the loss of plexinD1 protein, D1ThyCKO thymic cellularity was identical to that of the WT, indicating that loss of plexinD1 does not impact overall thymocyte expansion (Figure 1C). Further, the major $\mathrm{CD}^{-} \mathrm{CD}^{-}$double negative $(\mathrm{DN}), \mathrm{CD}^{+} \mathrm{CD} 8^{+} \mathrm{DP}$, and $\mathrm{CD}^{+}{ }^{+}$and $\mathrm{CD}^{+}{ }^{+}$single positive (SP) thymocyte subpopulations (defined by CD4 and CD8 expression) as well as progression through the DN1-4 stages (defined by CD44 and CD25 expression) as shown in Figure 1D were equivalent in D1ThyCKO and WT mice. Given that Plxnd1 gene transcripts and plexinD1 protein expression appear at the DP thymocyte level, it is of particular note that the steady state expansions of DP and SP thymocytes in both mice are comparable.

As shown in Figure S1B in Supplementary Material, CD69 ${ }^{+}$ expression was indistinguishable on WT and D1ThyCKO DP cells, suggesting that an equivalent number of thymocytes had undergone positive selection. In addition, the capacity of DP thymocytes to undergo apoptosis induced by anti-CD3 $\varepsilon \mathrm{mAb}$ in vitro was identical (Figure S1A in Supplementary Material). Further, there were no differences between WT and D1ThyCKO in transwell migration assays responding to CXCL12 and CCL25 (Figure S2 in Supplementary Material). Thus, by analysis of comparative phenotype, signaling, and response to chemokines in vitro, WT and D1ThyCKO thymocytes appeared indistinguishable. Using global thymic transcriptome analysis, we observed that the only gene downregulated in D1ThyCKO thymocytes more than 10 -fold is Plxnd1 itself (data not shown). Accordingly, we attribute effects observed in the subsequent thymic translocation of D1ThyCKO cells in vivo during thymic organogenesis to loss of thymocyte plexinD1.

\section{DP THYMOCYTE PLEXIND1 EXPRESSION REGULATES CORTICAL TO MEDULLARY TRANSLOCATION AFTER TCR ENGAGEMENT}

We then ascertained whether the $\mathrm{CD}^{+} 9^{+}$post-selection D1ThyCKO thymocytes exhibited the same cortical retention pattern as we had observed previously using germline Plxnd1 $1^{-1-}$ progenitors to repopulate Ly5 congenic animals post-transplant (15). Cortex and medulla were identified using anti-CD4 and anti-CD8 mAbs to define the medullary CD4 SP and CD8 SP thymocytes as well as merged CD4/CD8 signals to define cortical DP thymocytes (Figure 2). This delineation in the WT thymus was confirmed by the strong medullary expression of MHC class II and further confirmed by the medullary location for the ERTR5 marker $(29,30)$. Of note, in the WT thymus the CD69 ${ }^{+}$ post-selection thymocytes exhibit a medullary location while in the D1ThyCKO thymus, the $\mathrm{CD} 69^{+}$thymocytes are distributed 

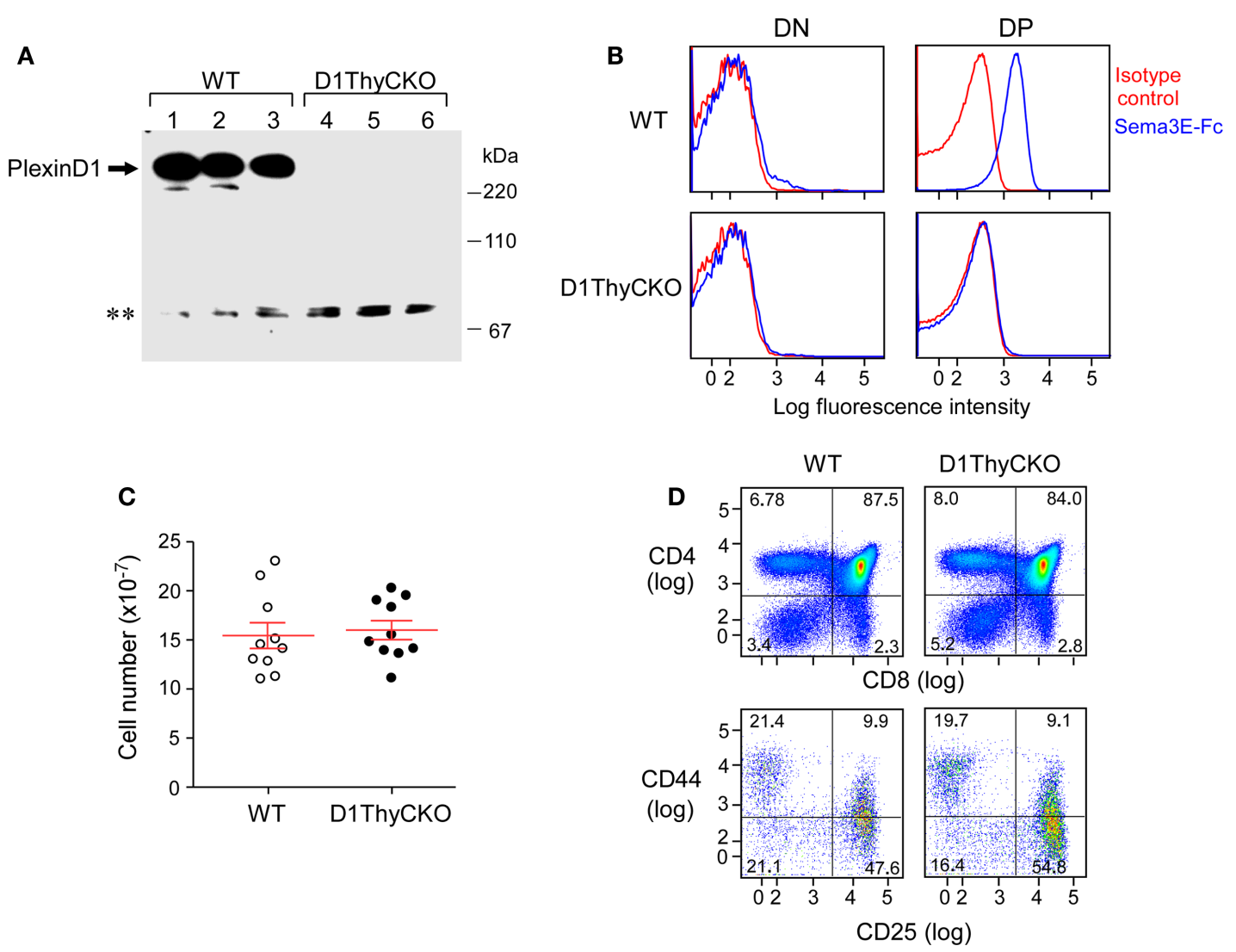

FIGURE 1 | PlexinD1 is not expressed in D1ThyCKO thymocytes but developmental progression is normal. (A) PlexinD1 was detected in total thymocyte lysates from 3- to 4-week-old WT or D1ThyCKO mice (three mice/strain) by Western blotting. The lower bands $\left({ }^{* *}\right)$ are non-specific bands common to both WT and D1ThyCKO thymocyte extracts and do not represent plexinD1 (representative of four independent experiments). (B) PlexinD1 detected by binding of sema3E-Fc (blue line) or control lgG2c (red line) after gating DP, CD4SP, CD8SP, and DN populations based on CD4 and CD8 $\alpha$

not only in the medulla but also throughout the cortex. This phenotype is distinct from other lineage Plxnd1 CKO mice to be described subsequently. Moreover, the ratio of medullarylocalized to cortical-localized $\mathrm{CD} 69^{+}$post-selection thymocytes was enumerated (Figure 3A), confirming the cortical retention D1ThyCKO thymus.

These results independently confirmed our original observation that plexinD1 expression in developing thymocytes plays an important regulatory role in the cortical to medullary translocation of differentiating thymocytes following engagement of their TCRs by self-pMHC on TEC. However, in contrast to the results obtained with germline Plxnd1 KO progenitor transplant reconstitution of the thymus, in the D1ThyCKO mice there is a completely normal thymic cortex and medullary demarcation on histological analysis (Figure 3B, upper panels) although at the level of CD4 and CD8 display, the separation between cortical DP and medullary SP thymocytes was less-well delineated, perhaps consequent to the differentiation within the cortex of mislocalized $\mathrm{CD} 9^{+}$cells (Figure 2 ), as previously observed with the germline expression (representative of six independent experiments). (C) Total thymocytes/thymus from WT or D1ThyCKO animals ( $n=10$ for each strain; $P=0.737$, not significant). (D) Thymocyte differentiation in WT and D1ThyCKO mice. The percentage representations of DN $\left(\mathrm{CD} 4^{-} \mathrm{CD} 8^{-}\right), \mathrm{DP}\left(\mathrm{CD} 4^{+} \mathrm{CD} 8^{+}\right)$, $\mathrm{CD} 4 \mathrm{SP}\left(\mathrm{CD}^{+} \mathrm{CD}^{-}\right)$, and $\mathrm{CD} 8 \mathrm{SP}\left(\mathrm{CD} 4^{-} \mathrm{CD}^{+}\right)$are depicted (upper panels). The percentages of DN1 (CD44 $\left.4^{\text {hi }} \mathrm{CD} 25^{\text {lo }}\right), \mathrm{DN} 2\left(\mathrm{CD} 44^{\mathrm{hi}} \mathrm{CD} 25^{\mathrm{hi}}\right), \mathrm{DN} 3$ $\left(\mathrm{CD} 44^{\text {lo }} \mathrm{CD} 25^{\mathrm{hi}}\right)$, and DN4 $\left(\mathrm{CD} 44^{\circ \mathrm{l}} \mathrm{CD} 25^{\text {lo }}\right.$ ) are shown (lower panels). Results represent six independent experiments.
Plxnd1 KO mice (15). Furthermore, of note, the ER-TR5 and MHCII markers also disengage from the dominantly medullary location in WT thymus and now track with the cortically localized $\mathrm{CD} 9^{+}$thymocytes in D1ThyCKO thymus (Figure 2). Quantitation of the medullary to cortical ratio for ER-TR5 in wt thymus $(2.97 \pm 0.35$; Figure S4 in Supplementary Material) supports its utilization as a relative medullary marker; likewise, the medullary to cortical ratio for MHC class II $(8.37 \pm 0.82)$ also supports its utilization as a medullary marker. In the D1ThyCKO thymus, however, the medullary to cortical ratio for both ERTR5 and MHC class II was significantly reduced on comparison with wt thymus $(1.39 \pm 0.14$ and $2.58 \pm 0.54$, respectively; Figure S4 in Supplementary Material). UEA1, also considered a medullary marker (31), does not segregate with ER-TR5 and MHCII in the D1ThyCKO mice (Figure S3 in Supplementary Material), instead remaining medullary in location. The alteration in ER-TR5 and MHCII distribution in association with $\mathrm{CD} 69^{+}$ thymocytes suggests possible crosstalk between developing thymocytes and TEC. Interestingly, this disengagement of ER-TR5 

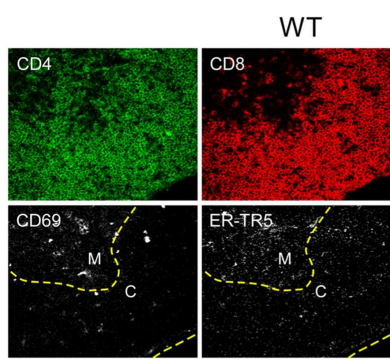

\section{D1EpCKO}
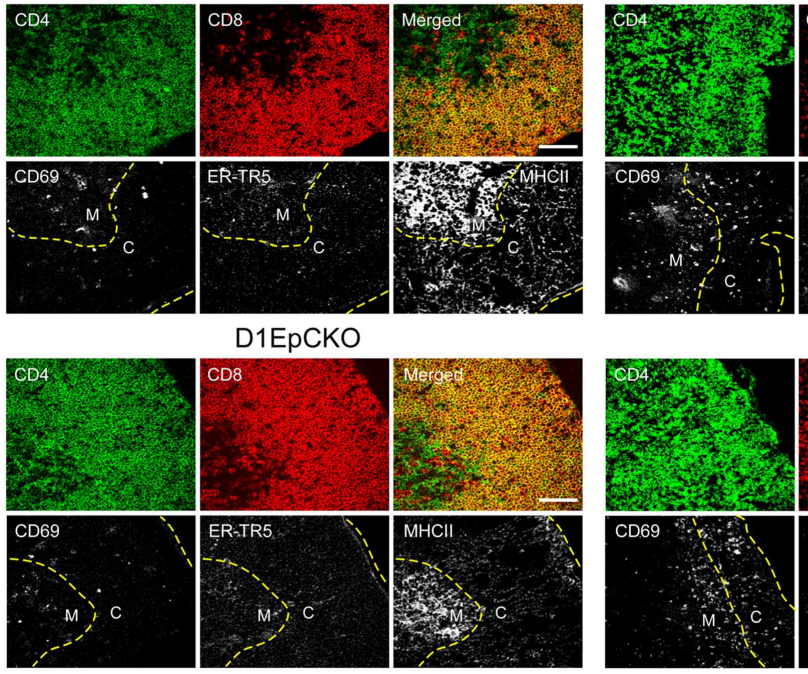

D1ThyCKO
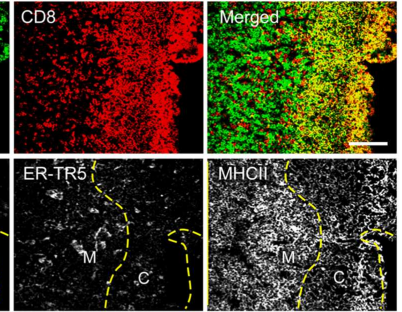

Sema3e $\%$
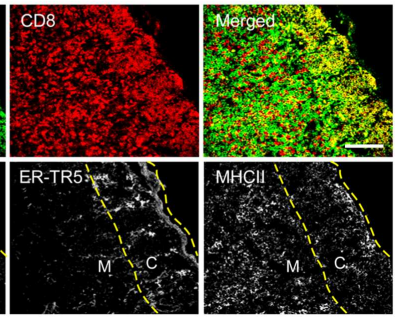

FIGURE 2 | $\mathrm{CD}^{+} 9^{+}$thymocytes are retained in the cortex in D1ThyCKO and Sema3e $e^{-1-}$ thymus but track to the medulla in WT and D1EpCKO mice. For each strain, thymic frozen sections were stained for CD4 (green) and CD8 (red) expression where the DP cells in the cortical regions appear yellow in merged images due to the co-expression of CD4 and CD8. The merged yellow DP signal was used to delineate the cortex (C) from the individual SP red and green signals in the medulla (M) and a yellow dashed line is inserted to indicate the apparent corticomedullary junction. A second yellow dashed line indicates the capsule position. CD69 is an activation marker expressed by positively selected thymocytes following TCR stimulation. ER-TR5 is considered a medullary marker (29) while MHCII $(\mathrm{I}-\mathrm{A} / \mathrm{I}-\mathrm{E})$ is preferentially expressed in medulla (30). Each figure is representative of three independent experiments. White bar in the merged images is $100 \mu \mathrm{m}$.
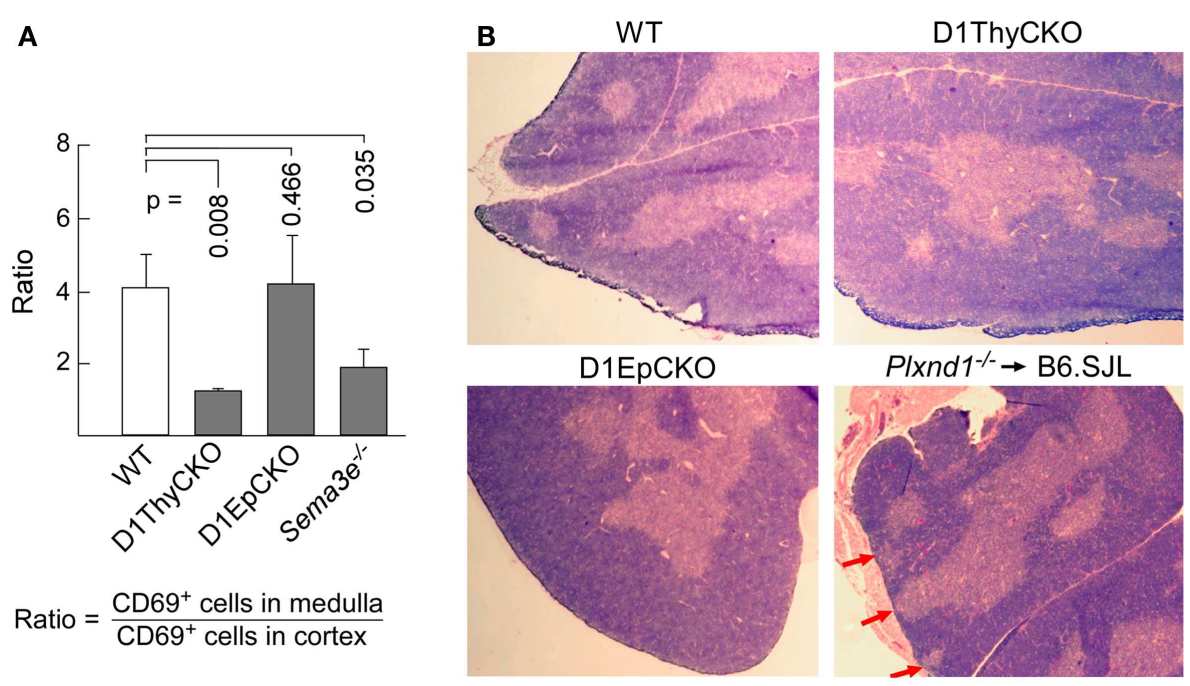

FIGURE 3 | Loss of plexinD1 disrupts cortical to medullary translocation of $\mathrm{CD} \mathbf{9}^{+}$DP thymocytes. (A) The regional distribution of $\mathrm{CD}_{69}{ }^{+}$cells in five separate frozen sections for each strain was determined based on FITC signal intensity within the regions defined by the apparent corticomedullary junction. After subtracting background signal, the ratio of fluorescence signal strength between the cortex and the medulla was calculated. A value $>1$ indicates stronger medullary presence on comparison with cortex. Error bars represent SEM. (B) Medullary structures were visualized by hematoxylin and eosin (H\&E) staining of WT, D1ThyCKO, and D1EpCKO thymi as well as in thymus in irradiated animals repopulated with $P / x n d 1^{-1-}$ fetal liver. The red arrows in the Plxnd1 $1^{-I_{-}}$section indicate regions of fused thymic medulla and capsule. Each figure is representative of three independent experiments. from medullary structures and association with $\mathrm{CD} 69^{+}$cortically located DP thymocytes is recapitulated in the developing thymus of Sema3e $e^{-1-}$ mice (Figure 2). Hence, this phenotype is a result of the direct disruption of sema3E-plexinD1 signaling in thymocytes, since plexinD1 is normally expressed in the Sema3e-null tissues (32). 


\section{WHILE EPITHELIAL SPECIFIC PIxnD1 CKO (D1EpCKO) IS WITHOUT THYMIC PHENOTYPE, TRANSIENT STIMULATION OF IMMATURE (PRE-SP) THYMOCYTES ALTERS TEC DEVELOPMENTAL PROGRAMING AND INITIATES MEDULLARY FORMATIONS}

Since D1ThyCKO lacked any medullary abnormality detected by histology, we next examined whether loss of plexinD1 in the epithelial cell population may account for the aberrant medullary phenotype observed by germline $P l x n d 1^{-1-}$ progenitor repopulation of B6.SJL thymi. Here we used pKer14-Cre ${ }^{+}$ Plxnd $1^{\text {flox/flox }}$ (D1EpCKO) mice where activation of $p$ Ker 14 during the bipotential TEC progenitor stage (33) will lead to deletion of Plxnd1 in descendant cTEC and mTEC. We found that the D1EpCKO mice were viable and exhibited normal thymic development and organization (Figure 2), histology (Figure 3B), and thymocyte development (data not shown). The ratio of medullary to cortical CD $69^{+}$thymocytes was $\sim 4$, directly comparable to that for WT CD69 ${ }^{+}$thymocytes migrating to the medulla (Figure 3A). Reinforcing the observed normal medullary development, in contrast to what was observed in D1ThyCKO mice, the ER-TR5 signal in D1EpCKO thymus localized with UEA1 in medullary regions comparable to that observed in WT thymus (Figure S3 in Supplementary Material). Thus epithelial loss of plexinD1 neither affected cortical to medullary migration of $\mathrm{CD} 9^{+}$thymocytes nor medullary development.

Our results imply that $\mathrm{CD} 69^{+}$thymocytes are responsible for the development of ER-TR5 ${ }^{+}$thymic signal and that ER-TR5 is a marker linked to $\mathrm{CD}^{+} 9^{+}$thymocyte position as opposed to medullary locale per se. This further implies that maturing DP thymocytes following TCR stimulation may play a role in initiating medullary development, a function previously ascribed exclusively to mature SP T cells (34). It has been reported previously that expression of $\alpha \beta$ TCR transgenes, even on a non-selecting background, may induce ER-TR5 ${ }^{+}$medulla development suggesting a role for $\alpha \beta$ TCR induction of thymic medullary structures in the absence of TCR activation (35). Since $T c r b^{+/+} R_{a g} 2^{-/-}$mice do not develop SP thymocytes or thymic medullary structures $(36,37)$, constitutive pre-TCR expression is insufficient for induction of medulla. In such mice, $\mathrm{T}$ cell development is arrested at the pre-TCR DP stage, but we reasoned that the pre-TCR and/or incomplete surface CD3 heterodimers (38) on these thymocytes might be stimulated via intravenous administration of anti-CD3 $\varepsilon$ $\mathrm{mAb}$ (clone 145.2C11) that rapidly enters the thymus (39).

To determine whether the DP thymocytes could be activated and induce medullary development, anti-CD $3 \varepsilon$ antibody was injected into $\mathrm{Tcrb}{ }^{+/+} \mathrm{Rag} 2^{-/-}$mice and thymi were collected at 0 , 6,24 , and $72 \mathrm{~h}$ to assess early activation and medulla development. A final analysis was done at 3 weeks post-injection to determine persistence and/or evolution of phenotypic changes. As shown in Figure 4, by $24 \mathrm{~h}$ post-2C11 injection, distinct regions containing CD4 and CD8 SP thymocytes were observed with extensive representation of the ER-TR5 medullary marker, MHC class II expression and UEA1 expression together with increased representation of $\mathrm{CD} 69^{+}$thymocytes. By $72 \mathrm{~h}$, these developmental changes had led to clear histological delineation of cortical and medullary regions but the representation of SP cells was diminished. The majority of cells were DP thymocytes and the ER-TR5 signal was considerably weaker while the UEA $1^{+}$signal, correlating well with areas of poor DP thymocyte representation, became even stronger. This latter result suggests that ER-TR5 discriminates early signals delivered by $\mathrm{CD} 69^{+} \mathrm{DP}$ thymocytes from those inducing development of $\mathrm{UEA}^{+}$mTEC, as observed for the disengagement of ER-TR5 signal from that of UEA1 in the D1ThyCKO

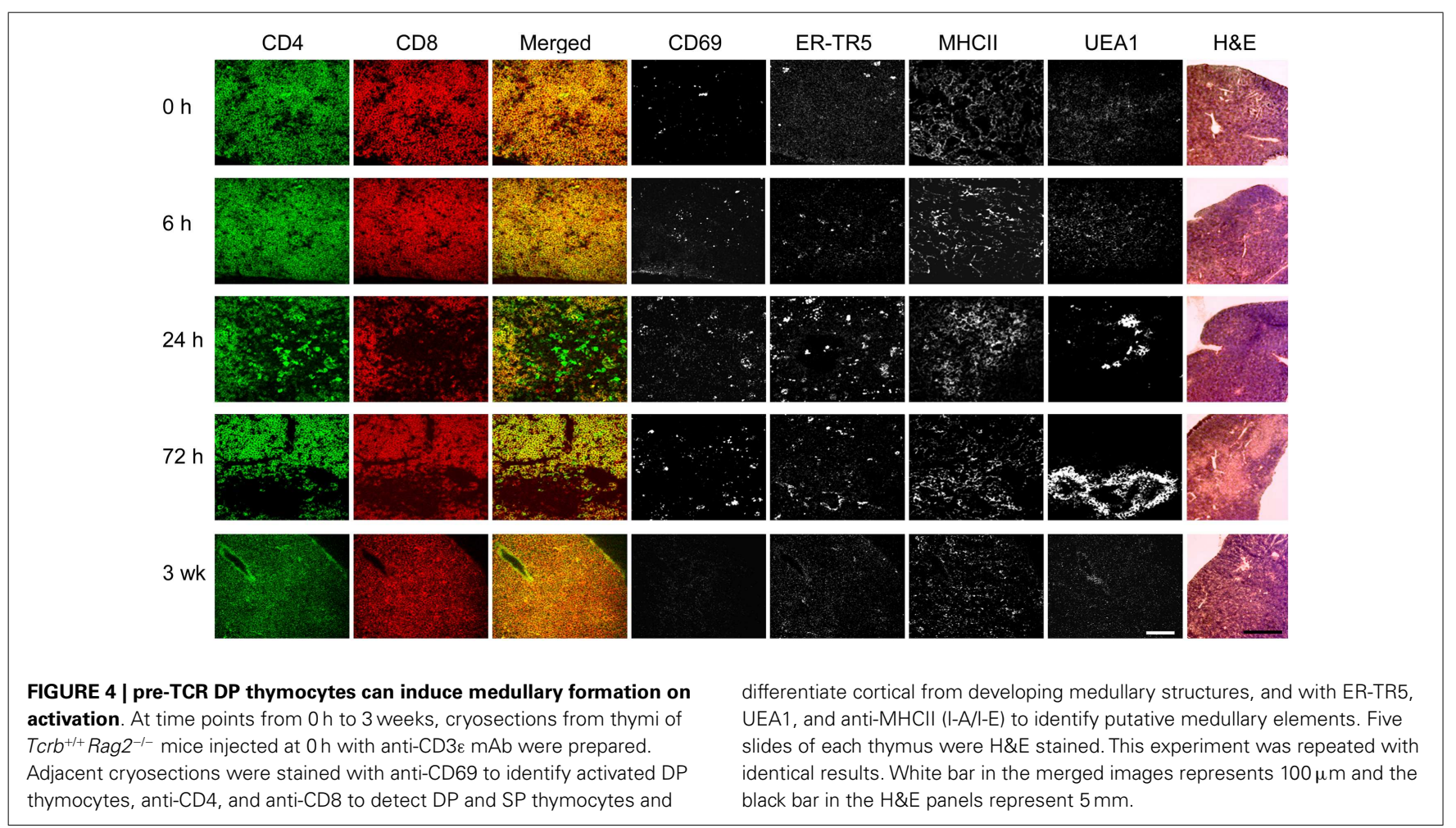


and Sema3e $e^{-1-}$ thymi where $\mathrm{CD} 69^{+}$thymocytes were retained in the cortex (Figure 2 and Figure S3 in Supplementary Material). In contrast, by 3 weeks after the single anti-CD $3 \varepsilon$ mAb injection, both ER-TR5 and UEA1 signals had diminished to almost undetectable levels. All indications of medullary formation had disappeared including histological demarcation of cortex and medulla, leading to a set of images indistinguishable from those at time $0 \mathrm{~h}$. Of note, in every instance, the developing corticomedullary junction was proximal to pre-existing larger vasculature defined by Endothelial cell Selective Adhesion Molecule (ESAM) as shown in Figure S5 in Supplementary Material (40). One possibility is that 2C11 distribution from the vasculature is greatest at these sites to most optimally trigger CD3 crosslinking on developing thymocytes.

\section{ENDOTHELIAL CELL PLEXIND1 EXPRESSION IMPACTS THYMIC MEDULLA LOCATION THROUGH REGULATION OF ANGIOGENESIS: ANALYSIS OF D1EnCKO MICE}

Although we had observed ectopic medullary formation in thymi of B6.SJL mice repopulated in vivo by $P l x n d 1^{-1-}$ fetal liver cells, no such abnormality was observed in D1ThyCKO or D1EpCKO mice where plexinD1 expression was specifically eliminated in thymocytes or epithelial cells, respectively. We concluded that plexinD1 must be operating in a distinct non-hematopoietic, non-epithelial lineage to impact thymic medullary developmental formation. In this regard, it is well established that within the fetal liver there are stem cells not only with lymphoid and myeloid potential but also capable of differentiating into hepatocytes and biliary epithelial cells $(25,41,42)$. Further, the fetal liver tissue expresses endothelial progenitor cells with angiogenic potential that may contribute to thymic vascular development $(23,24)$. Since plexinD1 and sema3E play a seminal role in controlling vascular endothelial branching during development, the possibility that $P l x n d 1^{-1-}$ endothelial stem cells delivered in the fetal liver inoculate may have influenced ectopic subcapsular medullary formation in our earlier report required further elucidation (15). To address whether plexinD1 expression on endothelial cells contributes to vasculature pattern formation, thereby supporting proper medullary formation, we crossed Plxnd $1^{\text {flox/flox }}$ mice and pTek(Tie2)-Cre transgenic mice expressing the Cre transgene in an endothelial-specific manner. The resulting $p$ Tek-Cre ${ }^{+} P l x n d 1^{\text {flox/flox }}$ (D1EnCKO) conditional mice manifest a defect in separation of the aorta and the pulmonary artery as previously reported and did not survive the neonatal period $(11,14)$. Furthermore, the thymic lobes failed to fuse, instead forming two separate thymic lobes on both sides of the truncus artery as we observed for germline Plxnd1-1mice (15).

To overcome the neonatal lethality of D1EnCKO mice, we transplanted excised fetal thymi under the renal capsule of WT B6.SJL recipient mice. This procedure allows the thymus carrying Plxnd1 $1^{-1-}$ endothelial cells to complete organogenesis (Figure 5A). After 3 weeks, the thymi with attached kidney were excised and cryosections prepared for histological analysis. The apposition of medulla to the thymic capsule was clearly observed histologically in the D1EnCKO thymus explant (Figure 5B). Analysis of sequential cryosections demonstrated that these medullary structures contained predominantly CD4 SP thymocytes and stained with the ER-TR5 medullary marker
(Figure 5B inset). This medullary structure is distinct from the cortical region otherwise marked by Tromal (anti-cytokeratin 8), a cTEC-expressed protein. In contrast, CD4 SP thymocytes are not present in the cortical subcapsular region of WT thymus where the merged signal of CD4 and CD8 on DP thymocytes dominated (Figures 2 and 5C).

To next determine whether these abnormal subcapsullary medullary formations were related to vascular development, we examined the expression of the vascular ESAM marker for significant differences between the WT and D1EnCKO thymi. In WT thymus, the ESAM signal was detected at highest levels on both sides of the corticomedullary junction with little representation in the outermost cortex (Figure 5C and Figure S6 in Supplementary Material). In contrast, in the D1EnCKO thymus the ESAM signal was broadly spread throughout the cortical region with strongest representation in the subcapsular area and less of a medullary presence (Figure 5C). The regulatory functions of plexinD1 for vascular endothelial branching are initiated by binding its ligand sema3E, leading us to examine whether a similar pattern of $\mathrm{ESAM}^{+}$vascular structures was present in the Sema $3 e^{-l-}$ thymus. As shown in Figure 6 and Figure S6 in Supplementary Material, unlike the pattern in the D1EnCKO thymus, the ESAM signal was broadly distributed throughout the Sema3e $e^{-1-}$ thymus without subcapsular representation implying that some functionality of plexinD1 in thymic vascular pattern formation is apparently sema3E-independent. Certainly, no difference was observed in the distribution of vascular $\mathrm{ESAM}^{+}$signal around the corticomedullary junction in WT and D1 ThyCKO thymus (Figure S6 in Supplementary Material), implying the ectopic medullary formations may be related to the effects of plexinD1 and sema3E upon thymic vascular bed development. Conversely, the distribution of $\mathrm{CD}^{+} 9^{+}$thymocytes in the D1EnCKO thymus clearly shows a strong medullary presence of $\mathrm{CD}^{+} 9^{+}$cells. Although there is collection of $\mathrm{CD} 69^{+}$cells around the corticomedullary junction, this did not translate into an overt retention of $\mathrm{CD}^{+}{ }^{+}$cells in the cortex (Figure S7 in Supplementary Material) and a normal medullary structure with appropriate localization of MHC class II, ER-TR5, and mTEC-expressed UEA1 and a clearly demarcated corticomedullary junction (Figure S7 in Supplementary Material). Consequently, loss of plexinD1 on the thymic endothelial cell population does not affect $\mathrm{CD}^{+} 9^{+}$thymocyte translocation from the cortex to the medulla but it does seem to alter cortical and medullary vascular development in a sema3E-independent manner.

To demonstrate that the development of D1EnCKO fetal thymus under recipient renal capsules properly represented the development ongoing in D1EnCKO, we analyzed thymi from newborn D1EnCKO mice using anti-Pecam1 (Platelet Endothelial Cell Adhesion Molecule-1, CD31) mAb that identifies vascular endothelial cells including those in newly formed vasculature (43) (Figure 5D and Figure S8 in Supplementary Material). In WT thymi the Pecam 1 signal was detected in a punctuate distribution throughout the thymus while in D1EnCKO thymi the Pecam 1 distribution was much more dense, with a $5.03 \pm 0.78$-fold increase in endothelial cell density over WT. Pecam 1 expression was particularly strong in cortical areas and the sections contained many ectopic medullary structures, several of which appeared to be 
A

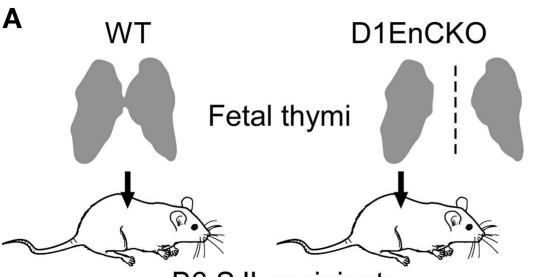

B6.SJL recipients

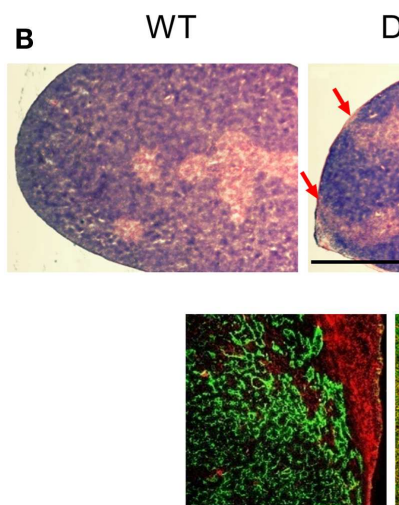

Troma1 ERT5
D1EnCKO
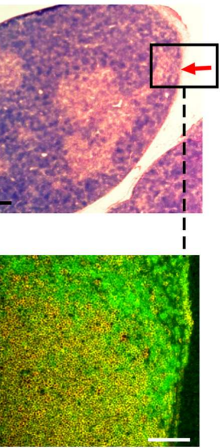

CD4 CD8
FIGURE 5 | PlexinD1 on thymic endothelial cells regulates the distribution of thymic medullary formations. (A) Schematic for analysis of D1EnCKO cells on thymus development. Fetal thymi harboring WT (both fused lobes transplanted) or D1EnCKO (single lobe transplanted) endothelial cells were allowed to develop under the recipient's renal capsule. All cell populations in the transplanted D1EnCKO thymus other than endothelial cells expressed plexinD1 appropriately as did all cells in the normal recipients. (B) Red arrows in H\&E stained panels indicate regions of medulla fused with the thymic capsule. A frozen section corresponding to the inset region in the D1EnCKO panel was stained for keratin 8 highly represented in cTEC using the Troma1 antibody $(56,57)$ and for ER-TR5 representing medullary development. Cortical-medullary demarcation was confirmed by the relative representation of merged $\mathrm{CD} 4^{+} \mathrm{CD} 8{ }^{+}$signal for DP cells and SP CD4 and CD8 cell development in

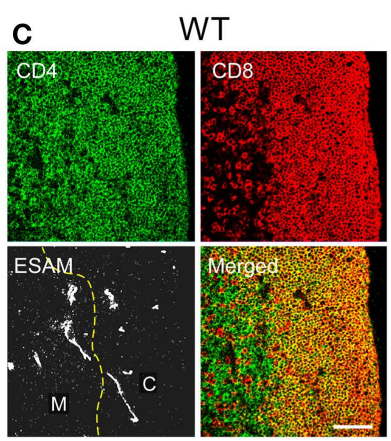

D1EnCKO

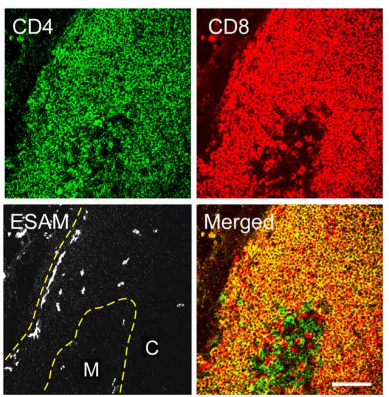

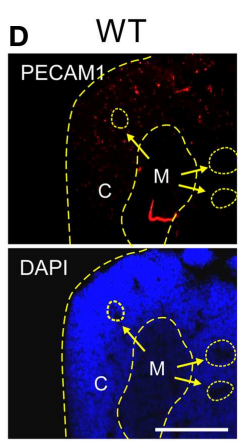

D1EnCKO

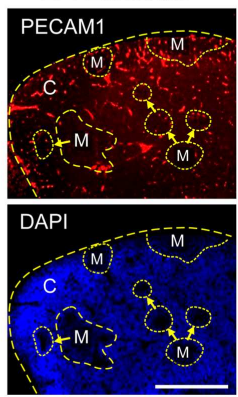

the subcapsular region. Result is representative of three independent transplantation experiments. White bar in the confocal image represents $100 \mu \mathrm{m}$ and in the H\&E stained image represents $5 \mathrm{~mm}$. (C) Cryosections of WT and D1EnCKO thymus were stained with anti-CD4-APC, anti-CD8 $\alpha$-TRITC, and anti-ESAM mAb-FITC. Dashed yellow line follows the corticomedullary junction; in the D1EnCKO panel, a second dashed yellow line to the left indicates the $\mathrm{ESAM}^{+}$cells in the subcapsullar zone. C, cortex; M, medulla. Result is representative of three independent transplantation experiments. White bar in the merged images are $100 \mu \mathrm{m}$ (D) Pecam1 staining to identify developing vascular endothelial structures. High density nuclear staining by DAPI delineates cortical regions and lower density nuclear staining correlates with medullary structures. C, cortex; M, medulla. The corticomedullary junctions and capsules are identified by dashed yellow lines. White bar in the images is $5 \mathrm{~mm}$. fused with the capsule. Thus the histological evidence for ectopic medullary formation in the Plxnd $1^{-1-}$ thymi can be definitively attributed to the expression of plexinD1 in thymic endothelial cells and their essential function in thymic vascular development.

\section{DISCUSSION}

Through cell-type specific deletion of plexinD1 during thymic organogenesis, we have dissected the involvement of plexinD1 and its ligand, sema3E, in thymic development dependent upon thymocyte, epithelial, and vascular endothelial cell crosstalk for proper organ pattern formation. We demonstrate that expression of plexinD1 during the DP stage of thymocyte differentiation is a necessary prerequisite for the orderly cortical to medullary translocation that follows positive selection. In the absence of thymocyte plexinD1, despite appropriate expression on all other cells in the developing thymus, the activated $\mathrm{CD} 9^{+}$cells do not translocate toward the medulla, instead largely remaining within the cortex. Further, those mislocalized $\mathrm{CD}^{+} 9^{+}$cells initiate developmental programing in local TEC cells in the cortex that induces the
ER-TR5 signal, a well characterized though molecularly undefined marker of the mTEC environment and used to define medullary locales. On the other hand, loss of plexinD1 in epithelial cells, either cTEC or mTEC, was unremarkable and hence of no consequence for any of the parameters monitored in the current study.

In contrast, loss of plexinD1 in the thymic endothelial progenitor population led to distinct reorganization of thymic vasculature that was associated with ectopic medullary formation in the cortex and a medulla topology characterized by fusion with the capsule. We observed this phenotype previously in thymi repopulated by Plxnd1 null cells (15). Thus thymic angiogenesis attributable to endothelial progenitors in these Plxnd1 ${ }^{-/-} \mathrm{CKO}$ mice and earlier germline $P l x n d 1^{-1-}$ KO progenitors determines the locale of medulla formation. We further show that currently accepted markers of mTEC are dynamically regulated since the cortically localized CD69 ${ }^{+}$DP thymocytes in D1ThyCKO were associated with increased cortical representation of ER-TR5 and MHCII markers, but not UEA1. The latter remained associated with the pre-formed, i.e., established, medullary structures. Finally, 

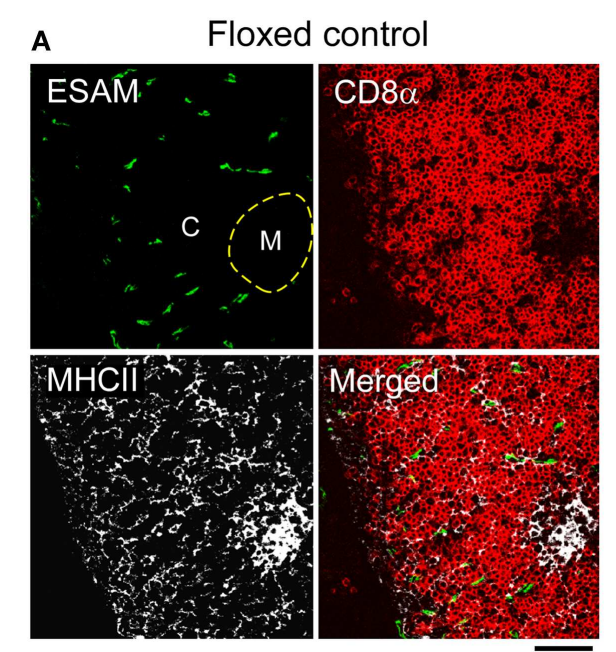

B

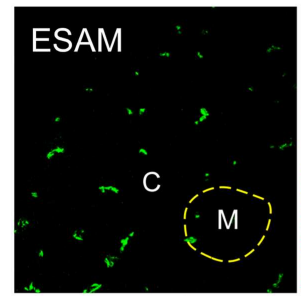

B6
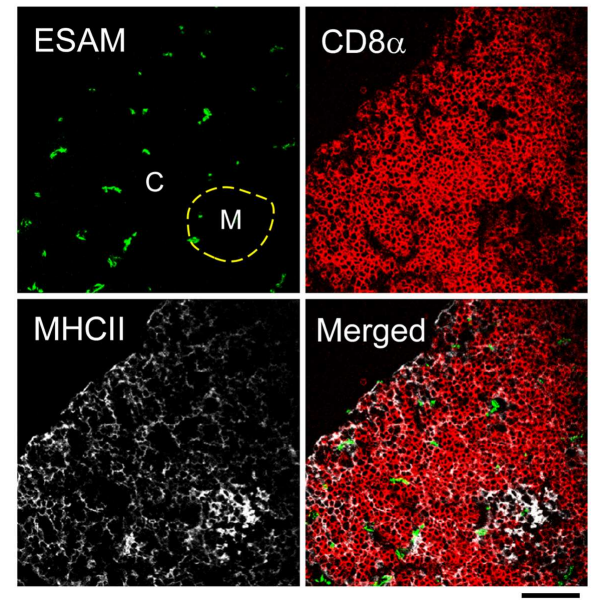

FIGURE 6 | Differential development of ESAM+ blood vessels in WT, D1EnCKO and Sema3e ${ }^{-/-}$thymi. Thymic cryosections of the indicated control mice and D1EnCKO (A) and Sema3e $e^{-/}$(B) mice were stained with anti-MHCII-APC, anti-CD8 $\alpha$-TRITC, and anti-ESAM mAb-FITC. Dashed line

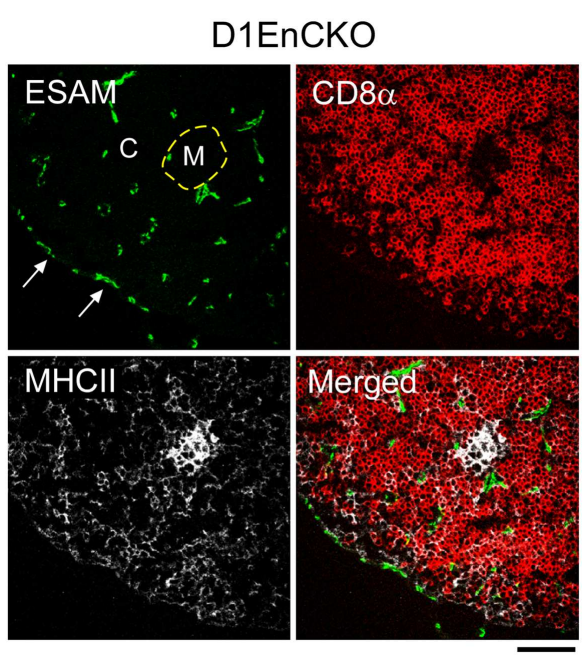

Sema3e-/-

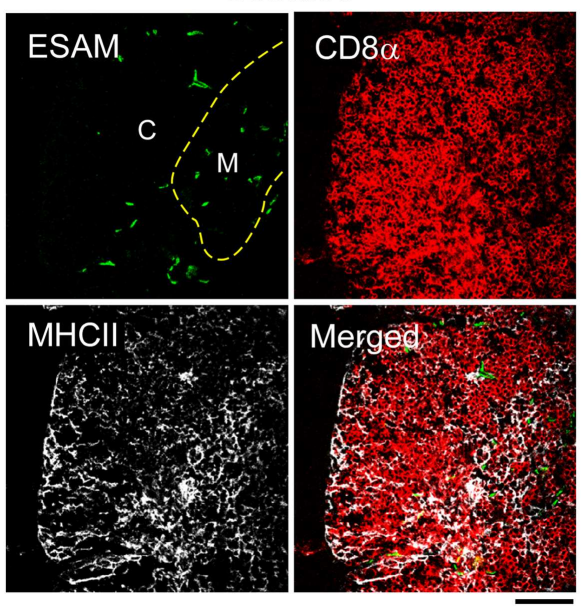

follows the corticomedullary junction. The result is representative of one of three independent experiments. The black bar below each merged image represents $100 \mu \mathrm{m}$ and is applicable to every panel in each block. C, cortex; $\mathrm{M}$, medulla. reinforcing the importance of the DP thymocytes in contributing to the initial although transient establishment of medullary formation, we demonstrate that DP activation in a $\mathrm{Crb}^{+/+} \mathrm{Rag}^{-1-}$ thymus results in development of medullary regions populated with SP thymocytes despite a genetic defect that precludes TCR expression and allows only pre-TCR to be displayed on the surface of DP thymocytes.

Our results clearly point to a dynamic interplay of thymocyte differentiation and vascular localization. Both processes are impacted by plexinD1 expression in independent cellular compartments, with consequent effects upon cTEC and mTEC function and medullary formation in turn effecting thymocyte differentiation. Since the thymocytes migrate along a path governed by differentiation-determined expression and function of chemokine receptors $(44,45)$, it is reasonable to conclude that blocks on maturation of thymocytes, exemplified here by loss of plexinD1, will lead to significant aberrations in thymus morphology. This notion is supported by results indicating that cTEC do not develop in mice where the DN1 $\rightarrow$ DN3 transition is defective (46) while a block on SP thymocyte development leads to absence of medullary formation, a state that can be altered by thymic influx of passively transferred SP T cells in SCID mice (34, 35). Collectively, such observations argue that mature SP cells are required for sustained medullary formation. In addition, we show here that anti-CD $3 \varepsilon \mathrm{mAb}$ triggered DP thymocytes can initiate differentiation of medullary structures, even if located in ostensibly "cortical" areas. Likewise, blocks in the orchestrated trafficking of differentiating thymocytes also may lead to aberrations in thymic morphology. For example, deficiency of CCR7 or of its ligands CCL19/CCL21 results in thymocytes maintaining a cortical position associated with ectopic medullary structure formation in the subcapsular zone and spontaneous autoimmunity with increased titers of circulating antibodies that lead to IgG deposition in renal glomeruli $(47,48)$. 
The DP thymocytes constitute the major population in the thymic cortex and we previously identified plexinD1 as a key regulator of DP thymocyte chemokine responses (15). In WT thymus repopulated by $P l x n d 1^{-1-}$ thymic precursors, we noted not only the cortical retention of $\mathrm{CD}^{+} 9^{+}$(activated) DP cells confirmed in this report in the D1ThyCKO mice, but also ectopic medullary formations lying under the capsule. In the context of plexinD1's key role in controlling vascular development and branching (11, 20), we investigated the possibility that the fetal liver containing early thymocyte progenitor cells used to repopulate the irradiated thymi contained $P l x n d 1^{-1-}$ endothelial precursors resulting in aberrant thymic vasculature formation. As noted previously, the development of the vascular bed in the thymus plays a key role in organizing the medullary epithelial cell compartment (49). Since D1EnCKO was neonatal lethal, we transplanted the D1EnCKO thymi under the recipient renal capsule to follow development. Under this circumstance, the abnormal fusion between thymic capsule and medulla was observed, demonstrating that blood vessel patterning directly affects the medulla location in thymus. Unlike the vasculature of the WT thymus, $\mathrm{ESAM}^{+}$blood vessels exist under the thymic capsule of D1EnCKO mice. In thymi lacking sema3E, however, a completely different phenotype was observed; $\mathrm{ESAM}^{+}$vessels were not observed under the thymic capsule but instead found broadly distributed throughout the thymic cortex.

This discordance suggests that either plexinD1 may respond to other thymic ligands distinct from sema3E, or that plexinD1 may contribute to intracellular regulatory pathways even in the absence of extracellular sema3E-mediated stimulation. In addition to the regulation of plexinD1 expression by VEGF mentioned above, the signaling for repulsion of developing neurites mediated by sema3E signaling through plexinD1 is mitigated or reversed by the ectodomain of neuropilin-1 (32). Of note, we observe no evidence for any direct interaction of neuropilin-1 ectodomain with either sema3E, plexinD1, or the sema3E-plexinD1 complex (data not presented), suggesting regulation of plexinD1 function by alternative signaling cascades mediated by Nrp1.

The apparently normal medullary structure and corticalmedullary demarcation in D1ThyCKO thymus by histological analysis in the context of a disorderly corticomedullary junction by confocal microscopy analysis point to a disengagement of medullary processes required for normal thymic function. In this report, we used ER-TR5 with or without UEA1 as another medullary marker in addition to MHC class II expression (2931, 50). In general, the MHC class II expression correlated well with that of ER-TR5. In the D1ThyCKO thymus, however, UEA1 remained associated with the histologically defined medulla while ER-TR5 uncoupled from this signal and localized with the cortically retained CD69+ DP thymocytes. Consequently, we propose that ER-TR5 is not a medullary marker but should be considered an epithelial cell marker induced by juxtaposition to developing activated T lymphoid cells. This scenario seems more probable than the suggestion that mTEC are rapidly migrating from the established medulla into the cortex, although such a possibility must be formally excluded in future studies.

An interesting corollary is that lack of plexinD1 on the DP thymocytes does not inhibit the differentiation of the thymocytes but only inhibits their migration. The involvement of plexinD1 as a regulator of migration rather than differentiation is supported strongly by similar functionality controlling migration not only of thymocytes but also B cells, macrophages, osteoblasts, prostate cancer cells, as well as colorectal tumor cell and melanoma cell line metastasis in models in vivo $(15,16,51-55)$. Control of migration has many facets, however, and may represent a regulation of adhesion through integrins rather than migration itself. Certainly, in transwell migration assays in the absence of potential interacting extracellular matrix components, we observe no effect of plexinD1 loss upon thymocyte responses to either CXCL12 or CCL25 (Figure S2 in Supplementary Material) and we have recently identified a direct role for plexinD1 independently as well as in a sema3E-dependent manner for regulating integrin adhesion (manuscript submitted). The additional role of plexinD1 in regulating thymic endothelial cell angiogenesis clearly has a direct impact upon the location of medullary formations. In this respect, it is of note that regional variations in thymic oxygenation are of critical importance for thymic structure as well as possible influence upon selection processes during thymocyte development (17-19).

\section{CONCLUSION}

The results presented herein represent a dissection of the simultaneous activities of the transmembrane receptor, plexinD1, functioning in disparate developmental processes mediated by different cellular compartments to regulate thymic organogenesis. PlexinD1 loss has a profound effect upon $\mathrm{CD} 9^{+}$positively selected DP thymocyte trafficking. The mislocalization of this thymocyte subset in the absence of gross alterations in thymic morphology impacts markers previously believed to delineate the medullary compartment. Meanwhile, loss of plexinD1 in vascular endothelial cells during thymic development leads to a distinct rearrangement of the thymic vascular structure that is clearly related to gross histological abnormalities in thymic morphology. In general, plexin signaling activity has been viewed as relatively passive in the absence of ligand. Comparison, however, of the thymus phenotypes of the WT, D1EpCKO, D1ThyCKO, and Sema3e $e^{-l-}$ null mice lays the groundwork for examination of plexinD1 signaling and functionality at the molecular level in both a sema3E ligand-independent and sema3E-dependent fashion.

\section{AUTHOR CONTRIBUTIONS}

Young I. Choi, Jing Tan, Jingang Gui, and Manvendra K. Singh performed all of the experiments. Young I. Choi, Jonathan S. Duke-Cohan, Jonathan A. Epstein, and Ellis L. Reinherz designed the experiments and analyzed the data. Jonathan S. Duke-Cohan, Young I. Choi, and Ellis L. Reinherz wrote the paper.

\section{ACKNOWLEDGMENTS}

We acknowledge the excellent technical assistance of Elizabeth Witten. This work was supported, in part, by National Institutes of Health Grants AI092378 to Young I. Choi and AI019807 to Ellis L. Reinherz and HL118768 to Jonathan A. Epstein.

\section{SUPPLEMENTARY MATERIAL}

The Supplementary Material for this article can be found online at http://www.frontiersin.org/Journal/10.3389/fimmu.2013.00392/ abstract 
Figure S1 shows that anti-CD3E-induced apoptosis is identical for WT and D1ThyCKO thymocytes, and that within the D1ThyCKO thymus, the number of developing CD69 ${ }^{+}$DP thymocytes is not significantly different to that in WT thymus thus any alteration in thymic localization represents a true positional shift in the DP population. Figure S2 depicts the identical responses of WT and D1ThyCKO cells to CXCL12 and CCL25 chemokines, supporting the proposal that mislocalization of the latter population is due to alteration in guidance cue signaling rather than an intrinsic defect in migratory capability. Figure S3 is a direct supplement to Figure 2, showing the distribution of the medullary UEA1 markers in the same thymic sections described in Figure 2. Figure S4 quantifies the disengagement of cortical ER-TR5 and MHC class II signal from canonical medullary structures observed in Figure 2. Figure S5 shows the development of transient medullary structures next to ESAM+ blood vessels in $T c r b^{+1+} R a g 2^{-1-}$ mice injected with anti-CD3 $\varepsilon$ mAb. Figure S6 reveals the disruption of association of $\mathrm{ESAM}^{+}$vessels with the corticomedullary junction in Sema3 $e^{-1-}$ thymus. Figure S7 shows normal cortical and medullary thymic marker localization in D1EnCKO thymus, even though some of the medullary structures may be embedded in the cortex and close to the capsule. Figure S8 demonstrates the dysregulation of vasculogenesis in D1EnCKO newborn thymus revealed by staining for endothelial cell Pecam1.

\section{Figure S1 | Plxnd1 deletion does not affect apoptosis of, or CD69} expression on, thymocytes following TCR stimulation. (A) One million total thymocytes were stimulated with plate-coated anti-CD3 $\mathrm{mAb}$ for the indicated time period. After incubation, the cells were harvested and stained with anti-CD4-APC/anti-CD8 $\alpha$-PE/anti-TCR $\beta$-FITC mAbs and analyzed by flow cytometry. The \%live DP population was normalized against \%live DP population at $0 \mathrm{~h}$. Error bars represent SEM. The result is representative of two independent experiments. (B) After stimulation of total thymocytes for the indicated time periods as in (A), the cells were harvested and stained with anti-CD4-APC/anti-CD8 $\alpha$-PE/anti-CD69-FITC mAbs and analyzed by flow cytometry. \%CD69+ DP thymocytes was calculated relative to total DP thymocytes. Error bars represent SEM. The result is representative of two independent experiments.

\section{Figure S2 | CXCL12- and CCL25-mediated migration is normal in} D1ThyCKO mice. Migration assays were performed with CXCL12 and CCL25 as described previously using both chemokines at $1 \mu \mathrm{g} / \mathrm{ml}$ (15). After the incubation for $2 \mathrm{~h}$, the cells migrating across the transwell membrane into the lower well were harvested and counted. White bars depict mock-treated (no chemokine) control experiments. Error bars represent SEM.

Figure S3 | UEA1 expression in plexinD1 conditional knockout and Sema3e $^{-1-}$ thymus. UEA1 expression in sections correlating to those depicted in Figure $\mathbf{2}$ in the main article. Note that the bulk of UEA1 signal is medullary in all cases. " $M$ " defines medullary area, " $C$ " defines cortical area; the dashed line represents the corticomedullary junction, and the solid line represents the thymic capsule.

Figure S4 | Quantitation of relative cortical and medullary ER-TR5 and MHC class II expression in wt and D1ThyCKO thymi. The relative signals for cortical and medullary-localized fluorescence were determined by image analysis of six D1ThyCKO thymic sections and nine wt sections. For each marker, the color image was converted to a grayscale image, six to seven regions without apparent signal were selected to represent background and the relative intensity for identically sized regions within areas demarcated as cortex or medulla were determined using the ImageJ java suite. The D1ThyCKO medullary $\mathrm{MHC}$ class II representation was significantly reduced on comparison with wt $(P<0.005)$ as was the medullary ER-TR5 representation $(P<0.02)$. Mean \pm SEM presented and compared using Student's $t$-test.

Figure S5 | Co-localization of ESAM ${ }^{+}$blood vessels and UEA1 ${ }^{+}$mTEC in thymi of $\mathbf{T r b}^{+/+}$Rag2 $^{-/-}$mice injected with anti-CD3 $\varepsilon$ mAb. Adjacent cryosections of each thymus were stained with the indicated Abs. anti-ESAM was used to visualize endothelial cells and UEA1 to visualize mTEC. The corticomedullary junction is indicated by the dashed line. The result represents two independent analyses. White bar in the ESAM panels is $100 \mu \mathrm{m}$ and applies to all panels.

Figure S6 | ESAM ${ }^{+}$blood vessel distribution is normal in D1ThyCKO mice. The thymic cryosections of the indicated mice were stained as described in Figure 5C. The result is representative of three independent experiments. The dashed line follows the corticomedullary junction where the demarcation between cortex and medulla is based on the location of DP and SP thymocytes, respectively. ESAM is an endothelial cell-specific marker. White bar in merged image is $100 \mu \mathrm{m}$. C, cortex; M, medulla.

Figure S7 | Normal CD69+ thymocyte distribution in transplanted D1EnCKO mice. Adjacent cryosections of D1EnCKO thymi transplanted under the B6.SJL renal kidney capsule were stained as described for Figure $\mathbf{2}$ and Figure S3. The cortex $(\mathrm{C})$ and the medulla $(\mathrm{M})$ were divided based on the location of DP and SP thymocytes. The left dashed line in each section follows the corticomedullary junction and the right dashed line depicts the thymic capsule. White bar in merged images is $100 \mu \mathrm{m}$. The figure is representative of two independent experiments. The graph depicts the enumeration of $\mathrm{CD} 69^{+}$cell density using the FITC signal intensity as described in Figure 3. Sections from three independent thymi were analyzed for each genotype (wt and D1EnCKO) and $P$ values were calculated using Student's $t$-test where the ordinate axis depicts the ratio of $\mathrm{CD}_{69} 9^{+}$cells in the medulla to $\mathrm{CD} 9^{+}$cells in the cortex. Error bars represent SEM. The $P$ value of 0.34 is not statistically significant.

Figure S8 | Pecam 1+ endothelial cells are increased in newborn D1EnCKO mice. (A) were collected from WT ( $n=2$, "Control" in figure) and D1EnCKO ( $n=3$, "Mutant" in figure) newborn mice and fixed overnight in 10\% formalin solution, then stained for Pecam1 (CD31). (B) The overall intensity/area was determined by image analysis for WT, and then both WT and D1EnCKO Pecam1 densities were expressed as a ratio of the WT values. Mean \pm SEM is depicted. The white bar, applicable to all panels, represents $2 \mathrm{~mm}$.

\section{REFERENCES}

1. Kruger RP, Aurandt J, Guan KL. Semaphorins command cells to move. Nat Rev Mol Cell Biol (2005) 6:789-800. doi:10.1038/nrm1740

2. Rohm B, Rahim B, Kleiber B, Hovatta I, Puschel AW. The semaphorin 3A receptor may directly regulate the activity of small GTPases. FEBS Lett (2000) 486:68-72. doi:10.1016/S0014-5793(00)02240-7

3. Oinuma I, Katoh H, Negishi M. Molecular dissection of the semaphorin 4D receptor plexin-B1-stimulated R-Ras GTPase-activating protein activity and neurite remodeling in hippocampal neurons. J Neurosci (2004) 24:11473-80. doi:10.1523/JNEUROSCI.3257-04.2004

4. Oinuma I, Ishikawa Y, Katoh H, Negishi M. The semaphorin 4D receptor Plexin-B1 is a GTPase activating protein for R-Ras. Science (2004) 305:862-5. doi:10.1126/science. 1097545

5. Tong Y, Chugha P, Hota PK, Alviani RS, Li M, Tempel W, et al. Binding of Racl, Rnd1, and RhoD to a novel Rho GTPase interaction motif destabilizes dimerization of the plexin-B1 effector domain. J Biol Chem (2007) 282:37215-24. doi:10.1074/jbc.M703800200

6. Wang Y, He H, Srivastava N, Vikarunnessa S, Chen YB, Jiang J, et al. Plexins are GTPase-activating proteins for Rap and are activated by induced dimerization. Sci Signal (2012) 5:ra6. doi:10.1126/scisignal.2002636

7. Halloran MC, Wolman MA. Repulsion or adhesion: receptors make the call. Curr Opin Cell Biol (2006) 18:533-40. doi:10.1016/j.ceb.2006.08.010

8. Oinuma I, Katoh H, Negishi M. Semaphorin 4D/Plexin-B1-mediated R-Ras GAP activity inhibits cell migration by regulating beta(1) integrin activity. J Cell Biol (2006) 173:601-13. doi:10.1083/jcb.200508204

9. Sakurai A, Jian X, Lee CJ, Manavski Y, Chavakis E, Donaldson J, et al. Phosphatidylinositol-4-phosphate 5-kinase and GEP100/Brag2 protein mediate 
antiangiogenic signaling by semaphorin 3E-plexin-D1 through Arf6 protein. J Biol Chem (2011) 286:34335-45. doi:10.1074/jbc.M111.259499

10. Watakabe A, Ohsawa S, Hashikawa T, Yamamori T. Binding and complementary expression patterns of semaphorin $3 \mathrm{E}$ and plexin D1 in the mature neocortices of mice and monkeys. J Comp Neurol (2006) 499:258-73. doi:10.1002/cne.21106

11. Gitler AD, Lu MM, Epstein JA. PlexinD1 and semaphorin signaling are required in endothelial cells for cardiovascular development. Dev Cell (2004) 7:107-16. doi:10.1016/j.devcel.2004.06.002

12. Torres-Vazquez J, Gitler AD, Fraser SD, Berk JD, Van NP, Fishman MC, et al. Semaphorin-plexin signaling guides patterning of the developing vasculature. Dev Cell (2004) 7:117-23. doi:10.1016/j.devcel.2004.06.008

13. Kanda T, Yoshida Y, Izu Y, Nifuji A, Ezura Y, Nakashima K, et al. PlexinD1 deficiency induces defects in axial skeletal morphogenesis. J Cell Biochem (2007) 101:1329-37. doi:10.1002/jcb.21306

14. Zhang Y, Singh MK, Degenhardt KR, Lu MM, Bennett J, Yoshida Y, et al. Tie2Cremediated inactivation of plexinD1 results in congenital heart, vascular and skeletal defects. Dev Biol (2009) 325:82-93. doi:10.1016/j.ydbio.2008.09.031

15. Choi YI, Duke-Cohan JS, Ahmed WB, Handley MA, Mann F, Epstein JA, et al. PlexinD1 glycoprotein controls migration of positively selected thymocytes into the medulla. Immunity (2008) 29:888-98. doi:10.1016/j.immuni.2008.10. 008

16. Holl EK, O’Connor BP, Holl TM, Roney KE, Zimmermann AG, Jha S, et al. Plexin-D1 is a novel regulator of germinal centers and humoral immune responses. J Immunol (2011) 186:5603-11. doi:10.4049/jimmunol.1003464

17. Hale LP, Braun RD, Gwinn WM, Greer PK, Dewhirst MW. Hypoxia in the thymus: role of oxygen tension in thymocyte survival. Am J Physiol Heart Circ Physiol (2002) 282:H1467-77. doi:10.1152/ajpheart.00682.2001

18. Biju MP, Neumann AK, Bensinger SJ, Johnson RS, Turka LA, Haase VH. Vhlh gene deletion induces Hif-1-mediated cell death in thymocytes. Mol Cell Biol (2004) 24:9038-47. doi:10.1128/MCB.24.20.9038-9047.2004

19. Bryson JL, Griffith AV, Hughes B III, Saito F, Takahama Y, Richie ER, et al. Cellautonomous defects in thymic epithelial cells disrupt endothelial-perivascular cell interactions in the mouse thymus. PLoS One (2013) 8:e65196. doi:10.1371/ journal.pone.0065196

20. Gu C, Yoshida Y, Livet J, Reimert DV, Mann F, Merte J, et al. Semaphorin 3E and plexin-D1 control vascular pattern independently of neuropilins. Science (2005) 307:265-8. doi:10.1126/science.1105416

21. Kim J, Oh WJ, Gaiano N, Yoshida Y, Gu C. Semaphorin 3E-Plexin-D1 signaling regulates VEGF function in developmental angiogenesis via a feedback mechanism. Genes Dev (2011) 25:1399-411. doi:10.1101/gad.2042011

22. Zygmunt T, Gay CM, Blondelle J, Singh MK, Flaherty KM, Means PC, et al. Semaphorin-PlexinD1 signaling limits angiogenic potential via the VEGF decoy receptor sFlt1. Dev Cell (2011) 21:301-14. doi:10.1016/j.devcel.2011.06.033

23. Shi Q, Rafii S, Wu MH, Wijelath ES, Yu C, Ishida A, et al. Evidence for circulating bone marrow-derived endothelial cells. Blood (1998) 92:362-7.

24. Purhonen S, Palm J, Rossi D, Kaskenpaa N, Rajantie I, Yla-Herttuala S, et al. Bone marrow-derived circulating endothelial precursors do not contribute to vascular endothelium and are not needed for tumor growth. Proc Natl Acad Sci US A (2008) 105:6620-5. doi:10.1073/pnas.0710516105

25. Chen Q, Khoury M, Limmon G, Choolani M, Chan JK, Chen J. Human fetal hepatic progenitor cells are distinct from, but closely related to, hematopoietic stem/progenitor cells. Stem Cells (2013) 31:1160-9. doi:10.1002/stem.1359

26. Allen JM, Forbush KA, Perlmutter RM. Functional dissection of the lck proximal promoter. Mol Cell Biol (1992) 12:2758-68.

27. Cheng J, Turksen K, Yu QC, Schreiber H, Teng M, Fuchs E. Cachexia and graftvs.-host-disease-type skin changes in keratin promoter-driven TNF alpha transgenic mice. Genes Dev (1992) 6:1444-56. doi:10.1101/gad.6.8.1444

28. Fadel BM, Boutet SC, Quertermous T. Functional analysis of the endothelial cellspecific Tie2/Tek promoter identifies unique protein-binding elements. Biochem $J$ (1998) 330(Pt 1):335-43.

29. Small M, Van Ewijk W, Gown AM, Rouse RV. Identification of subpopulations of mouse thymic epithelial cells in culture. Immunology (1989) 68:371-7.

30. Surh CD, Gao EK, Kosaka H, Lo D, Ahn C, Murphy DB, et al. Two subsets of epithelial cells in the thymic medulla. J Exp Med (1992) 176:495-505. doi:10.1084/jem.176.2.495

31. Farr AG, Anderson SK. Epithelial heterogeneity in the murine thymus: fucose-specific lectins bind medullary epithelial cells. J Immunol (1985) 134: 2971-7.
32. Chauvet S, Cohen S, Yoshida Y, Fekrane L, Livet J, Gayet O, et al. Gating of Sema3E/PlexinD1 signaling by neuropilin-1 switches axonal repulsion to attraction during brain development. Neuron (2007) 56:807-22. doi:10.1016/j. neuron.2007.10.019

33. Bleul CC, Corbeaux T, Reuter A, Fisch P, Monting JS, Boehm T. Formation of a functional thymus initiated by a postnatal epithelial progenitor cell. Nature (2006) 441:992-6. doi:10.1038/nature04850

34. Surh CD, Ernst B, Sprent J. Growth of epithelial cells in the thymic medulla is under the control of mature T cells. J Exp Med (1992) 176:611-6. doi:10.1084/ jem.176.2.611

35. Shores EW, Van Ewijk W, Singer A. Maturation of medullary thymic epithelium requires thymocytes expressing fully assembled CD3-TCR complexes. Int Immunol (1994) 6:1393-402. doi:10.1093/intimm/6.9.1393

36. Shinkai Y, Rathbun G, Lam KP, Oltz EM, Stewart V, Mendelsohn M, et al. RAG-2-deficient mice lack mature lymphocytes owing to inability to initiate V(D)J rearrangement. Cell (1992) 68:855-67. doi:10.1016/0092-8674(92) 90029-C

37. van Ewijk W, Shores EW, Singer A. Crosstalk in the mouse thymus. Immunol Today (1994) 15:214-7. doi:10.1016/0167-5699(94)90246-1

38. Levelt CN, Mombaerts P, Iglesias A, Tonegawa S, Eichmann K. Restoration of early thymocyte differentiation in T-cell receptor beta-chain-deficient mutant mice by transmembrane signaling through CD3 epsilon. Proc Natl Acad Sci US A (1993) 90:11401-5. doi:10.1073/pnas.90.23.11401

39. Fiorini E, Schmitz I, Marissen WE, Osborn SL, Touma M, Sasada T, et al. Peptideinduced negative selection of thymocytes activates transcription of an NF-kappa B inhibitor. Mol Cell (2002) 9:637-48. doi:10.1016/S1097-2765(02)00469-0

40. Hirata K, Ishida T, Penta K, Rezaee M, Yang E, Wohlgemuth J, et al. Cloning of an immunoglobulin family adhesion molecule selectively expressed by endothelial cells. J Biol Chem (2001) 276:16223-31. doi:10.1074/jbc.M100630200

41. Barcena A, Galy AH, Punnonen J, Muench MO, Schols D, Roncarolo MG, et al. Lymphoid and myeloid differentiation of fetal liver CD34+lineage-cells in human thymic organ culture. J Exp Med (1994) 180:123-32. doi:10.1084/ jem.180.1.123

42. Strain AJ, Crosby HA, Nijjar S, Kelly DA, Hubscher SG. Human liver-derived stem cells. Semin Liver Dis (2003) 23:373-84. doi:10.1055/s-2004-815563

43. Baldwin HS, Shen HM, Yan HC, Delisser HM, Chung A, Mickanin C, et al. Platelet endothelial cell adhesion molecule-1 (PECAM-1/CD31): alternatively spliced, functionally distinct isoforms expressed during mammalian cardiovascular development. Development (1994) 120:2539-53.

44. Zuniga-Pflucker JC. T-cell development made simple. Nat Rev Immunol (2004) 4:67-72. doi:10.1038/nri1257

45. Halkias J, Melichar HJ, Taylor KT, Ross JO, Yen B, Cooper SB, et al. Opposing chemokine gradients control human thymocyte migration in situ. J Clin Invest (2013) 123:2131-42. doi:10.1172/JCI67175

46. Klug DB, Carter C, Gimenez-Conti IB, Richie ER. Cutting edge: thymocyteindependent and thymocyte-dependent phases of epithelial patterning in the fetal thymus. J Immunol (2002) 169:2842-5.

47. Kurobe H, Liu C, Ueno T, Saito F, Ohigashi I, Seach N, et al. CCR7-dependent cortex-to-medulla migration of positively selected thymocytes is essential for establishing central tolerance. Immunity (2006) 24:165-77. doi:10.1016/j. immuni.2005.12.011

48. Davalos-Misslitz AC, Rieckenberg J, Willenzon S, Worbs T, Kremmer E, Bernhardt G, et al. Generalized multi-organ autoimmunity in CCR7-deficient mice. Eur J Immunol (2007) 37:613-22. doi:10.1002/eji.200636656

49. Anderson M, Anderson SK, Farr AG. Thymic vasculature: organizer of the medullary epithelial compartment? Int Immunol (2000) 12:1105-10. doi:10. 1093/intimm/12.7.1105

50. Muller SM, Terszowski G, Blum C, Haller C, Anquez V, Kuschert S, et al. Gene targeting of VEGF-A in thymus epithelium disrupts thymus blood vessel architecture. Proc Natl Acad Sci U S A (2005) 102:10587-92. doi:10.1073/pnas. 0502752102

51. Casazza A, Finisguerra V, Capparuccia L, Camperi A, Swiercz JM, Rizzolio S, et al. Sema3E-Plexin D1 signaling drives human cancer cell invasiveness and metastatic spreading in mice. J Clin Invest (2010) 120:2684-98. doi:10.1172/JCI42118

52. Blanc V, Nariculam J, Munson P, Freeman A, Klocker H, Masters J, et al. A role for class 3 semaphorins in prostate cancer. Prostate (2011) 71:649-58. doi:10.1002/pros.21281 
53. Hughes A, Kleine-Albers J, Helfrich MH, Ralston SH, Rogers MJ. A class III semaphorin (Sema3e) inhibits mouse osteoblast migration and decreases osteoclast formation in vitro. Calcif Tissue Int (2012) 90:151-62. doi:10.1007/s00223-0119560-7

54. Movassagh H, Shan L, Halayko AJ, Roth M, Tamm M, Chakir J, et al. Neuronal chemorepellent Semaphorin 3E inhibits human airway smooth muscle cell proliferation and migration. J Allergy Clin Immunol (2013). doi:10.1016/j.jaci.2013. 06.011

55. Wanschel A, Seibert T, Hewing B, Ramkhelawon B, Ray TD, Van Gils JM, et al. Neuroimmune guidance cue Semaphorin $3 \mathrm{E}$ is expressed in atherosclerotic plaques and regulates macrophage retention. Arterioscler Thromb Vasc Biol (2013) 33:886-93. doi:10.1161/ATVBAHA.112.300941

56. Kemler R, Brulet P, Schnebelen MT, Gaillard J, Jacob F. Reactivity of monoclonal antibodies against intermediate filament proteins during embryonic development. J Embryol Exp Morphol (1981) 64:45-60.

57. Klug DB, Carter C, Crouch E, Roop D, Conti CJ, Richie ER. Interdependence of cortical thymic epithelial cell differentiation and T-lineage commitment. Proc Natl Acad Sci U S A (1998) 95:11822-7. doi:10.1073/pnas.95.20.11822
Conflict of Interest Statement: The authors declare that the research was conducted in the absence of any commercial or financial relationships that could be construed as a potential conflict of interest.

Received: 11 October 2013; paper pending published: 01 November 2013; accepted: 07 November 2013; published online: 19 November 2013.

Citation: Choi YI, Duke-Cohan JS, Tan J, Gui J, Singh MK, Epstein JA and Reinherz EL (2013) Plxnd1 expression in thymocytes regulates their intrathymic migration while that in thymic endothelium impacts medullary topology. Front. Immunol. 4:392. doi: 10.3389/fimmu.2013.00392

This article was submitted to T Cell Biology, a section of the journal Frontiers in Immunology.

Copyright (c) 2013 Choi, Duke-Cohan, Tan, Gui, Singh, Epstein and Reinherz. This is an open-access article distributed under the terms of the Creative Commons Attribution License (CC BY). The use, distribution or reproduction in other forums is permitted, provided the original author(s) or licensor are credited and that the original publication in this journal is cited, in accordance with accepted academic practice. No use, distribution or reproduction is permitted which does not comply with these terms. 